\title{
Striated Myogenesis and Peristalsis in the Fetal Murine Esophagus Occur without Cell Migration or Interstitial Cells of Cajal
}

\author{
M. Rishniw P.J. Fisher R.M. Doran S.P. Bliss M.I. Kotlikoff \\ Department of Biomedical Sciences, College of Veterinary Medicine, Cornell University, Ithaca, N.Y., USA
}

\section{Key Words}

Transdifferentiation $\cdot$ Smooth muscle actin $\cdot$ Organ culture

\begin{abstract}
Esophageal striated myogenesis progresses differently from appendicular myogenesis, but the mechanism underlying this process is incompletely understood. Early theories of transdifferentiation of smooth muscle into striated muscle are not supported by transgenic fate-mapping experiments; however, the origin of esophageal striated muscle remains unknown. To better define the process of striated myogenesis, we examined myogenesis in murine fetal cultured esophageal whole-organ explants. Embryonic day 14.5 (E14.5) esophagi maintained a functional contractile phenotype for up to 7 days in culture. Striated myogenesis, as evidenced by myogenin expression, proceeded in a craniocaudal direction along the length of the esophagus. Esophageal length did not change during this process. Complete, but not partial, mechanical disruption of the rostral esophagus inhibited myogenesis distally. Addition of fibroblast growth factor-2 (FGF-2) to the culture media failed to inhibit striated myogenesis, but attenuated smooth muscle actin expression and reduced peristaltic activity. Inhibition of c-kit failed to inhibit peristalsis. These results suggest that striated myogenic precursors are resident along the entire length of the
\end{abstract}

esophagus by day 14.5 and do not migrate along the esophagus after E14.5. Induction of myogenesis craniocaudally appears to require physical continuity of the esophagus and is not inhibited by FGF-2. Finally, peristalsis in E14.5 esophagi appears not to be regulated by interstitial cells of Cajal.

Copyright $\odot 2008$ S. Karger AG, Basel

\section{Abbreviations used in this paper}

E9-10 embryonic day 9-10

eGFP enhanced green fluorescent protein

ERK extracellular signal-regulated kinase

FGF-2 fibroblast growth factor 2 (basic fibroblast growth

factor)

ICC interstitial cells of Cajal

MEK MAP-ERK kinase

NFDM nonfat dry milk

SDS sodium dodecyl sulfate

SMC smooth muscle cells

SMCG2 smooth-muscle MHC-driven Cre recombinase/

eGFP transgenic mice

StMC striated muscle cells

TBS Tris-buffered saline

TBST Tris-buffered saline Tween 20 buffer

\section{KARGER}

๑ 2008 S. Karger AG, Basel

Fax +41613061234

E-Mail karger@karger.ch

www.karger.com
Accessible online at: www.karger.com/cto
Dr. Michael I. Kotlikoff

Department of Biomedical Sciences

College of Veterinary Medicine, Cornell University

S2005 Schurman Hall, Ithaca, NY 14853-6401 (USA)

Tel. +1 607253 3771, Fax +1 607253 3701, E-Mail mik7@cornell.edu 


\section{Introduction}

Esophageal striated myogenesis is a complex process that differs from striated myogenesis elsewhere in the body. The muscularis externa of the murine esophagus initially develops as a smooth muscle tube, apparent by embryonic day 9-10 (E9-10), but then transforms into an almost exclusively striated muscle tube over the following 3 weeks [Zhao and Dhoot, 2000a-c; Rishniw et al., 2003; Wörl and Neuhuber, 2005; Rishniw et al., 2007].

Approximately $50 \%$ of this smooth-to-striated muscle transformation occurs postnatally [Rishniw et al., 2007]. Although initially hypothesized to occur via transdifferentiation of smooth muscle cells (SMCs) into striated muscle cells (StMCs) [Patapoutian et al., 1995; Kablar et al., 2000; Stratton et al., 2000], more recent studies have provided compelling evidence that StMCs arise from a distinct and separate population of precursors [Zhao and Dhoot, 2000a-c; Rishniw et al., 2003; Wörl and Neuhuber, 2005; Rishniw et al., 2007]. Studies have shown three independent fates of esophageal SMCs: apoptosis [Wörl and Neuhuber, 2005], persistence within the striated muscle layer as remnant SMCs [Rishniw et al., 2007], and compaction into the lower esophageal sphincter [Rishniw et al., 2007].

The origin of StMCs and the means by which they populate the esophagus remain largely undetermined. Skeletal myoblasts could differentiate from pluripotent mesenchymal stem cells within the esophagus, although this seems unlikely given that other striated muscle precursors arise from neural crest cells that have migrated either to form somites or to other regions (e.g. facial muscles). Alternatively, neural crest cells similar to those that give rise to pharyngeal and lingual muscles could migrate to the esophagus during early esophageal development, forming a resident population of myogenic precursors. Lingual and pharyngeal striated muscles are apparent by E10.5, while esophageal StMC are not detected before E11.5-12.5, indicating that if simultaneous migration occurs, myodifferentiation must be slightly delayed relative to other nonsomatic muscles. Finally, it is possible that a later migration of precursors derived from somites or neural crest could occur, giving rise to striated myogenesis in a rostral to caudal pattern.

To address the myogenic potential of the embryonic mouse esophagus and further define this process, we examined striated myogenesis ex vivo. Using wild-type and SMCG2 transgenic fetuses, we cultured esophageal explants in toto for up to 7 days, and examined myogenesis and esophageal functional integrity by immunohisto- chemical, fluorescent microscopic and pharmacological means, using fibroblast growth factor (FGF-2) and neutralizing anti-c-kit antibodies to alter either myogenesis or peristalsis. Our study demonstrates that striated myogenesis occurs in the explanted E14.5 esophagus maintained in organ culture in a rostrocaudal direction and is dependent on the physical integrity of the esophagus and unaffected by exposure to FGF-2. Finally, we show that cultured fetal esophagi display prominent peristaltic contractions for up to 7 days in culture and this peristalsis is not dependent on interstitial cells of Cajal (ICC).

\section{Materials and Methods}

Organ Culture

Female C57BL/6J mice were mated with male SMCG2 ${ }^{+/ \mathrm{WT}}$ transgenic mice [these mice are maintained as hemizygous transgenic mice and express Cre recombinase and enhanced green fluorescent protein (eGFP) under the control of a smooth muscle myosin heavy chain promoter; thus the aforementioned mating would produce, on average, $50 \%$ wild-type fetuses, and $50 \%$ hemizygous transgenic fetuses] and euthanatized by $\mathrm{CO}_{2}$ asphyxiation 14.5 days postcoitus as approved by the Institutional Animal Care and Use Committee. Uteri were immediately excised and placed in sterile Petri dishes on ice, and fetuses removed and placed in ice-cold PBS ( $\mathrm{pH}$ 7.4) on ice. Fetal esophagi were explanted in toto, with tongue, pharynx/larynx and stomach attached in ice-cold PBS using a dissecting microscope (magnification 18-30×), transferred immediately onto 6.5 -mm-diameter 3 - $\mu \mathrm{m}$-pore-size polycarbonate membrane filters (Transwells, Corning, Inc., Corning, N.Y., USA), and incubated in $270-\mu$ l organ culture media (BGJb media, $10 \mathrm{mg} / \mathrm{ml}$ ascorbic acid, $20 \%$ heat-inactivated fetal calf serum, penicillin/streptomycin/amphotericin) at $37^{\circ} \mathrm{C}$ in $5 \% \mathrm{CO}_{2}$ for up to 7 days. Media were exchanged every $12 \mathrm{~h}$. Motility was assessed every $24 \mathrm{~h}$ by observing each of the esophagi in culture on an inverted dissecting scope for up to 2 min each and timing contraction intervals. For esophagi that were transected or partially transected, a razor blade was gently pressed across the esophagus approximately $25 \%$ of the length caudal to the pharynx. Disruption of muscular continuity was determined by loss of propagation of peristalsis across the disrupted region and loss of fluorescent continuity along the muscularis externa. Esophageal smooth muscle fluorescence (which provided an indirect measure of smooth muscle cellular differentiation) was examined with a dissecting microscope coupled to a magnesium arc lamp and a GFP filter.

To determine if striated myodifferentiation could be inhibited by a known inhibitor of myodifferentiations that acts through the MAP kinase pathway (basic FGF) [Tortorella et al., 2001], hrFGF2 (233-FB, R\&D Systems, Minneapolis, Minn., USA), MAP-ERK kinase (MEK) inhibitor PD98059 (513000, Calbiochem, La Jolla, Calif., USA) [Tortorella et al., 2001], or both were added to achieve final concentrations of $25 \mu \mathrm{g} / \mathrm{ml} \mathrm{hrFGF-2}$ and/or $50 \mu \mathrm{M}$ PD98059. Fresh hrFGF-2 and/or PD 98059 were added every $12 \mathrm{~h}$, when media were exchanged. To determine if fetal esophageal peristalsis was regulated by ICC, esophagi were cultured as described above, 
but with $5 \mu \mathrm{g} / \mathrm{ml}$ ACK2 antibody (Gibco BRL, Gaithersburg, Md., USA), which is a neutralizing antibody directed against c-kit (which, in turn, is essential in maintaining ICC phenotype), added to the media, and exchanged every $12 \mathrm{~h}$ [David et al., 2005].

Esophagi used for immunohistochemical analysis were fixed in the culture filters for $24 \mathrm{~h}$ in $10 \%$ formalin, mounted in specimen processing gel (Histogel, Richard Allan Scientific, Kalamazoo, Mich., USA) and then embedded in paraffin and sectioned at $4 \mu \mathrm{m}$.

We performed each series of esophageal culture experiments at least 6 times. For baseline culture experiments, all fetuses in a litter were used (8-12 fetuses/litter). When performing experiments with hrFGF-2 and/or PD98059, litters were divided into 3 groups, control group, hrFGF-2 group and PD98059/hrFGF-2 group, with an average of 3 fetuses per group. Thus each experimental condition was repeated on at least 18 fetuses.

We estimated the extent of myogenesis at each time point from composite digital images of esophagi that were obtained at identical magnifications. Using a calibrated scale bar, we were able to estimate the total esophageal length and the percentage of the esophagus displaying striated myogenesis at each time point.

\section{Immunohistochemistry}

For myogenin immunostaining, fixed sections were steamed for $15 \mathrm{~min}$ in $0.01 \mathrm{M}$ citrate buffer ( $\mathrm{pH}$ 6.0), blocked in mouse IgG blocking reagent (M.O.M., Vector Labs, Burlingame, Calif., USA) for $1 \mathrm{~h}$ with Tris-buffered saline (TBS)-Triton, $10 \%$ goat serum and $10 \%$ nonfat dry milk (NFDM) for $20 \mathrm{~min}$, and incubated at $37^{\circ} \mathrm{C}$ for $20 \mathrm{~min}$ and at room temperature for $40 \mathrm{~min}$ with mouse anti-rat myogenin (1:30, M3559, DakoCytomation, Carpinteria, Calif., USA). Sections were then incubated with biotinylated antimouse (M.O.M.) for $20 \mathrm{~min}$ at room temperature, followed by streptavidin-conjugated horse-radish peroxidase (Zymed Laboratories, San Francisco, Calif., USA) and visualized with AEC (Zymed Laboratories). For smooth muscle actin immunostaining, fixed sections were blocked in mouse IgG blocking reagent as above and incubated at $37^{\circ} \mathrm{C}$ for $20 \mathrm{~min}$ and at room temperature for 40 min with mouse-anti-human anti- $\alpha$-SM actin (1:60, M0851, clone 1A4, DakoCytomation). Secondary antibody staining was as above. To determine ACK2 antibody penetration into cultured organs, fixed slides were blocked for 20 min with $10 \%$ goat serum (Zymed Laboratories) and incubated with biotinylated goat antirat IgG (Jackson Immunoresearch Labs, Westgrove, Pa., USA) for $20 \mathrm{~min}$ at room temperature, followed by Texas Red-avidin DCS (Vector Labs).

Positive and negative controls were performed with each immunohistochemical experiment, using either smooth muscle tissue for anti- $\alpha$-SM actin or ACK2 antibodies and replacing these primary antibodies from the sections with mouse or rat IgG as a nonspecific negative control.

\section{Immunoblotting}

Esophagi cultured with FGF-2 and with FGF-2 plus PD98059 were disrupted by several freeze-thaw cycles in a radioimmunoprecipitation assay buffer containing $20 \mathrm{mM}$ Tris- $\mathrm{HCl}(\mathrm{pH} \mathrm{8.0)}$, $137 \mathrm{~mm} \mathrm{NaCl}, 10 \%$ glycerol, $1 \%$ Nonidet P-40, $0.1 \%$ sodium dodecyl sulfate (SDS), $0.5 \%$ deoxycholate, $2 \mathrm{~mm}$ EDTA, $5 \mathrm{~mm}$ sodium vanadate, $0.2 \mathrm{mM}$ phenylmethylsulfonyl fluoride, and $5 \mathrm{~mm}$ benzamidine. After centrifugation to remove cell debris, the protein concentrations in the whole-cell lysates were determined.
Proteins $(30 \mu \mathrm{g})$ from individual samples were resolved by SDSPAGE and transferred to polyvinylidene difluoride membranes by electroblotting. The blots were blocked with 5\% NFDM in TBS Tween 20 buffer (TBST; 10 mM Tris- $\mathrm{HCl}, \mathrm{pH}$ 7.5; $150 \mathrm{mM} \mathrm{NaCl}$; $0.05 \%$ Tween 20 ) and then probed with anti-phospho-ERK1/2 antibody (1:1,000 dilution; Cell Signaling, Beverly, Mass., USA) or anti-ERK antibody (1:500 dilution; Santa Cruz Biotechnology, Santa Cruz, Calif., USA). Species-specific secondary antibodies coupled to horseradish peroxidase (1:5,000 dilution in 5\% NFDM/ TBST; Santa Cruz Biotechnology) were used to detect the primary antibodies. Protein bands were visualized using enhanced chemiluminescence (PerkinElmer, Boston, Mass., USA).

\section{Results}

\section{Striated Myogenesis Occurs in Fetal Esophageal \\ Muscularis Externa in Culture}

E14.5 esophagi were explanted and immunostained for myogenin, a specific transcription factor involved in striated myogenesis, to examine the process of esophageal myogenesis. Over the 7-day period of examination, myogenin-positive cells first appeared in the rostral esophagus, with myogenin expression progressing caudally along the length of the esophagus over the 7-day period (fig. 1). Myogenin-positive cells could be detected along approximately $25-30,50$ and $70 \%$ of the esophagus after 3, 5 and 7 days of culture, respectively, but not at culture day 0 , consistent with previous observations [Zhao and Dhoot, 2000c]. We could not detect any myogenin-positive cells near the lower esophageal sphincter, in the stomach or in any esophageal layer other than the muscularis externa at any time, but myogenin-positive cells were detected in the pharynx and lingual musculature as early as culture day 0 (fig. 1). Myogenin expression appeared to progress slightly more rapidly in the external (longitudinal) layer of the muscularis externa than the internal layer. No differences were apparent between wild-type and SMCG2 $2^{+/ \mathrm{WT}}$ transgenic fetuses in any experiments, so results were pooled.

Fig. 1. Sagittal sections of murine esophagi from E14.5 fetuses show progressive myogenin expression over 7 days of culture: day 0 (A), day 5 (B) and day 7 (C). a Myogenin expression (red nuclear staining) in the diaphragm. $\mathbf{b}$ Myogenin expression in 3-4 subpharyngeal nuclei. c Robust myogenin expression in the tongue. $\mathbf{d}$ Myogenin expression extending caudally along the rostral muscularis externa layers. e Myogenin expression in the muscularis externa approaching the stomach. Scale bars for large panels $=500 \mu \mathrm{m}$; scale bars for insets $=100 \mu \mathrm{m}$. 


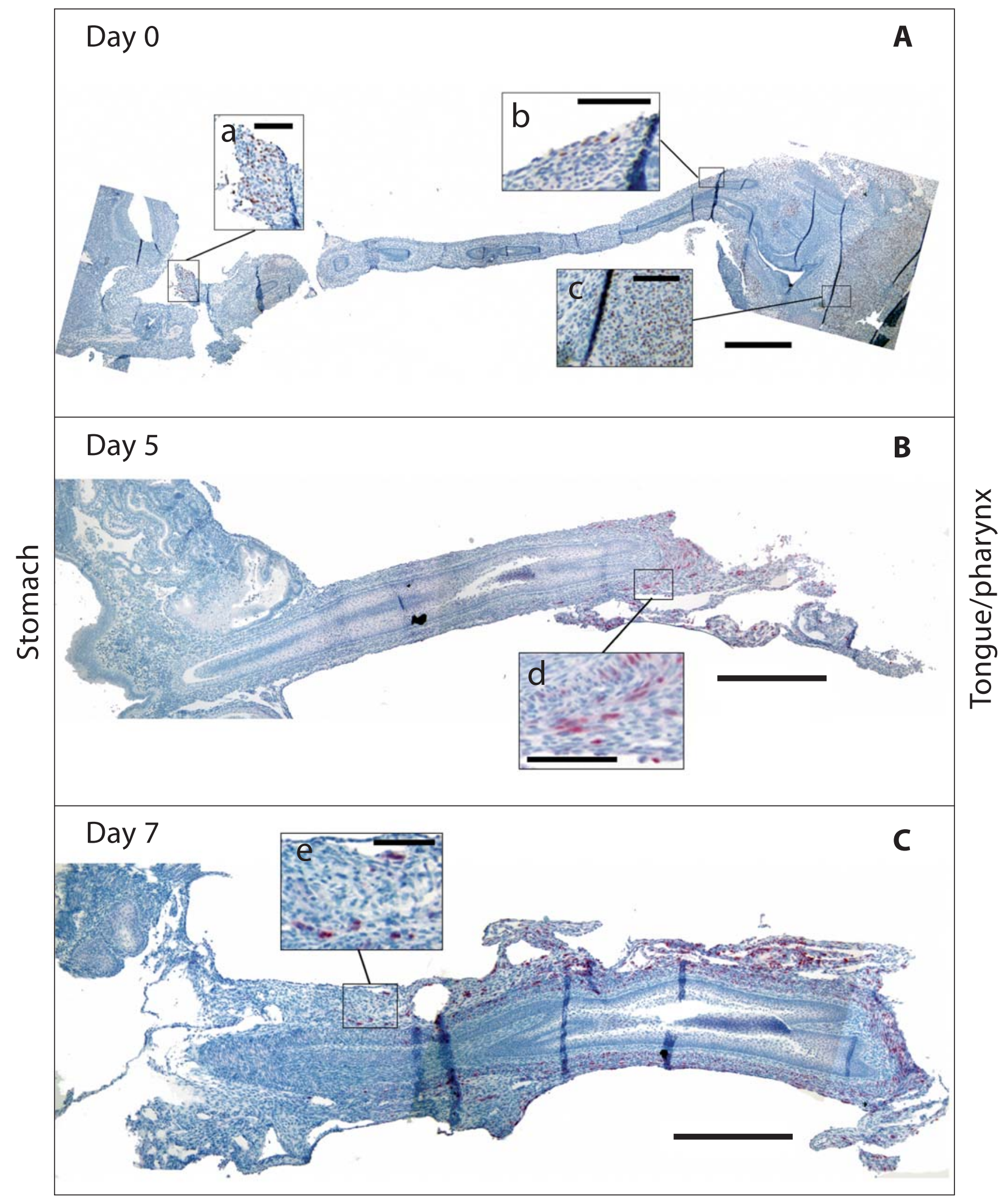




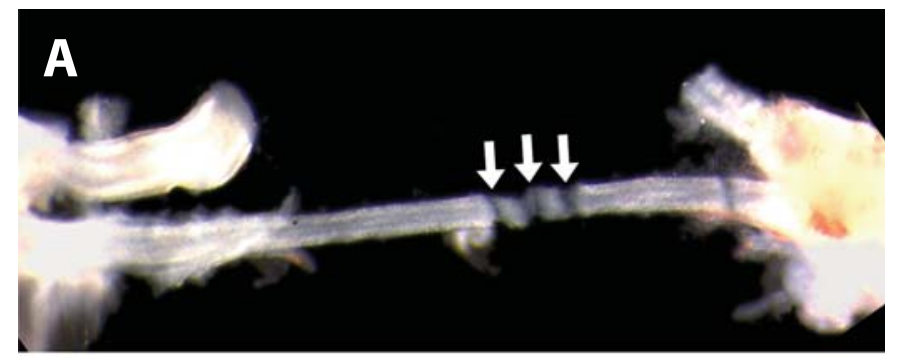

Stomach

Pharynx

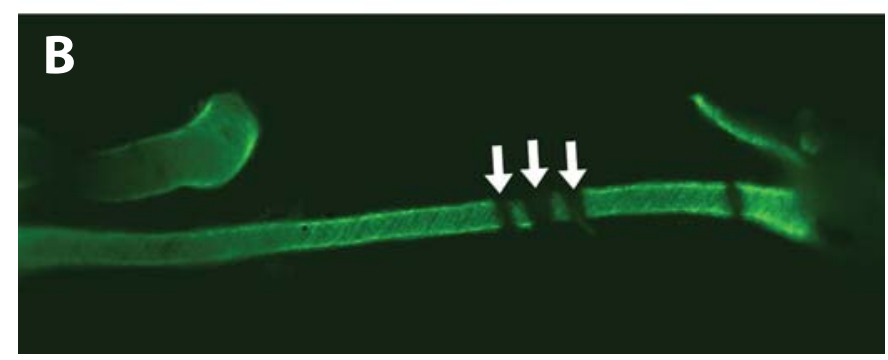

Fig. 2. Mechanical disruption of the esophageal muscularis externa results in discontinuity of smooth muscle fluorescence. A Fresh whole mount E14.5 esophagus explanted from SMCG2 transgenic mice (which express eGFP in smooth muscle) showing 3 adjacent zones of muscularis externa disruption (arrows). B Fluorescent imaging shows loss of smooth muscle fluorescence through the disrupted zones (arrows).

Striated Myogenic Precursors Are Resident within the Smooth Muscle Muscularis Externa

Our previous studies, and those of other investigators, have refuted the hypothesis that SMCs in the esophageal muscularis externa transdifferentiate into StMCs [Zhao and Dhoot, 2000a-c; Rishniw et al., 2003, 2007]; however, the origin of the StMCs remains unknown. Recently, investigators have suggested that esophageal StMCs arise from a resident population of precursor cells [Wörl and Neuhuber, 2005]. However, that study did not rule out the possibility that these striated muscle precursor cells actively migrate into the smooth muscle layers from a subpharyngeal location. To examine this hypothesis, we partially or completely transected fetal esophagi at harvest (E14.5) just caudal to the larynx/pharynx. In partially transected esophagi the serosal and mucosal layers remained intact, but the muscularis externa was completely separated (fig. 2A), as demonstrated by an interruption of fluorescence of the smooth muscle layers (fig. 2B) and a failure of propagation of peristaltic waves through this zone (online suppl. video 1, www.karger.com/doi/ $10.1159 / 000155225)$. If muscle cell precursors migrate caudally to their locations in the muscularis externa during development, we would expect to observe a lack of myogenin staining caudal to the partially transected zone, because the migratory path would be disrupted. However, we found robust myogenin expression in the caudal esophageal segment at culture day 7 (fig. 3A), indicating that striated muscle precursors are already resident within the developing muscularis externa at E14.5. On the other hand, in completely transected esophagi, where there was no serosal continuity (and therefore no proximity of the rostral and caudal esophageal segments), we could not detect striated myogenesis in the caudal esophageal segment, despite continued robust peristalsis of both segments, and myogenin expression in the proximal segment (fig. 3B). Together, these findings suggest that myogenic precursors do not migrate from the pharyngeal musculature at the time of myodifferentiation, but are resident in the proximal esophagus. Moreover, these results suggest that local factors are responsible for the activation of resident precursor cells, as segments that maintain close proximity show evidence of a progressive myodifferentiation. During striated myogenesis the smooth muscle component of the esophagus remains essentially unaltered, and remains considerably larger than the StMC component.

\section{Fetal Esophagi Remain Functional and Viable for up to 7 Days in Culture}

Esophageal explants maintained a functional phenotype for up to 7 days in culture. They failed to grow (length and width remained unchanged), but demonstrated vigorous spontaneous peristalsis throughout this period. Peristaltic waves occurred approximately every 20-30 s when examined at room temperature on an inverted dissecting scope, and propagated rostrally and caudally. Some of these waves were coordinated with gastric contractions, but not every gastric contraction propagated a retrograde peristaltic wave (online suppl. video 2, www.karger.com/doi/10.1159/000155225). Histologically, $\alpha$-smooth muscle actin could be identified in 3 distinct muscle layers along the length of the esophagus, corresponding to the 2 layers of the muscularis externa and the inner layer of the muscularis mucosa (fig. 4A), indicating that the SMCs maintained their phenotype throughout this period.

\section{Esophageal Striated Myogenesis Is Not FGF \\ Dependent}

Striated myogenesis is modulated by FGF-2, which inhibits the expression of the differentiation markers MyoD 


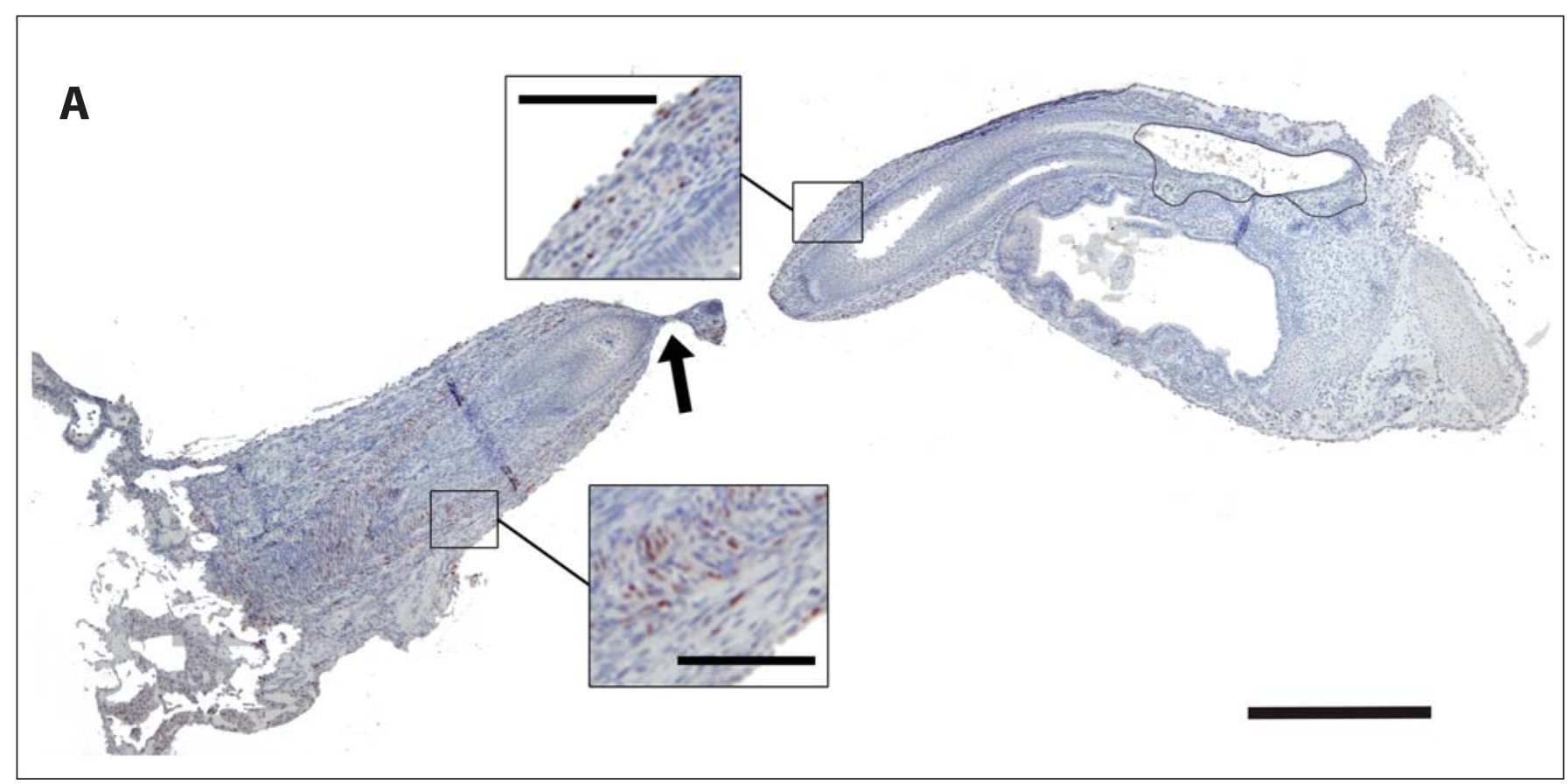

\section{Stomach}

Pharynx

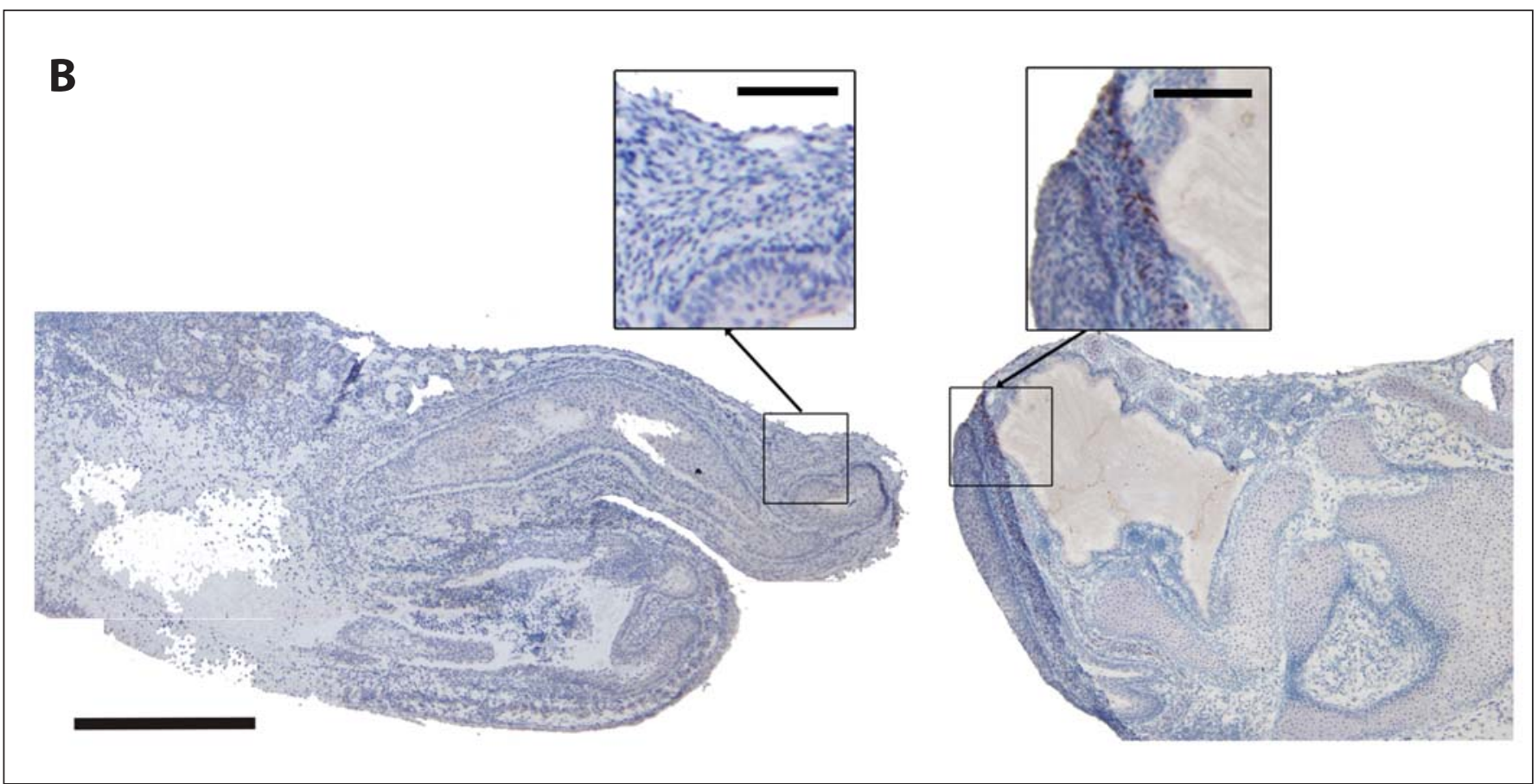

Fig. 3. Striated myogenesis occurs in esophagi with mechanically disrupted muscularis externa. A Sagittal section of E14.5 esophagus with a disrupted muscularis externa (arrow) but contiguity of the rostral and caudal segments after 7 days of culture shows myogenin expression on both sides of the zone of disruption (insets). The apparent dissociation to the right of the arrow (rostral) is a sectioning artifact, where a section of esophagus was positioned outside of the sectioning plane. B Myogenin expression is visible only in the subpharyngeal segment in a completely transected E14.5 esophagus after 7 days of culture, but is absent in the caudal segment (insets). Scale bars for large panels $=500 \mu \mathrm{m}$; scale bars for insets $=100 \mu \mathrm{m}$. 


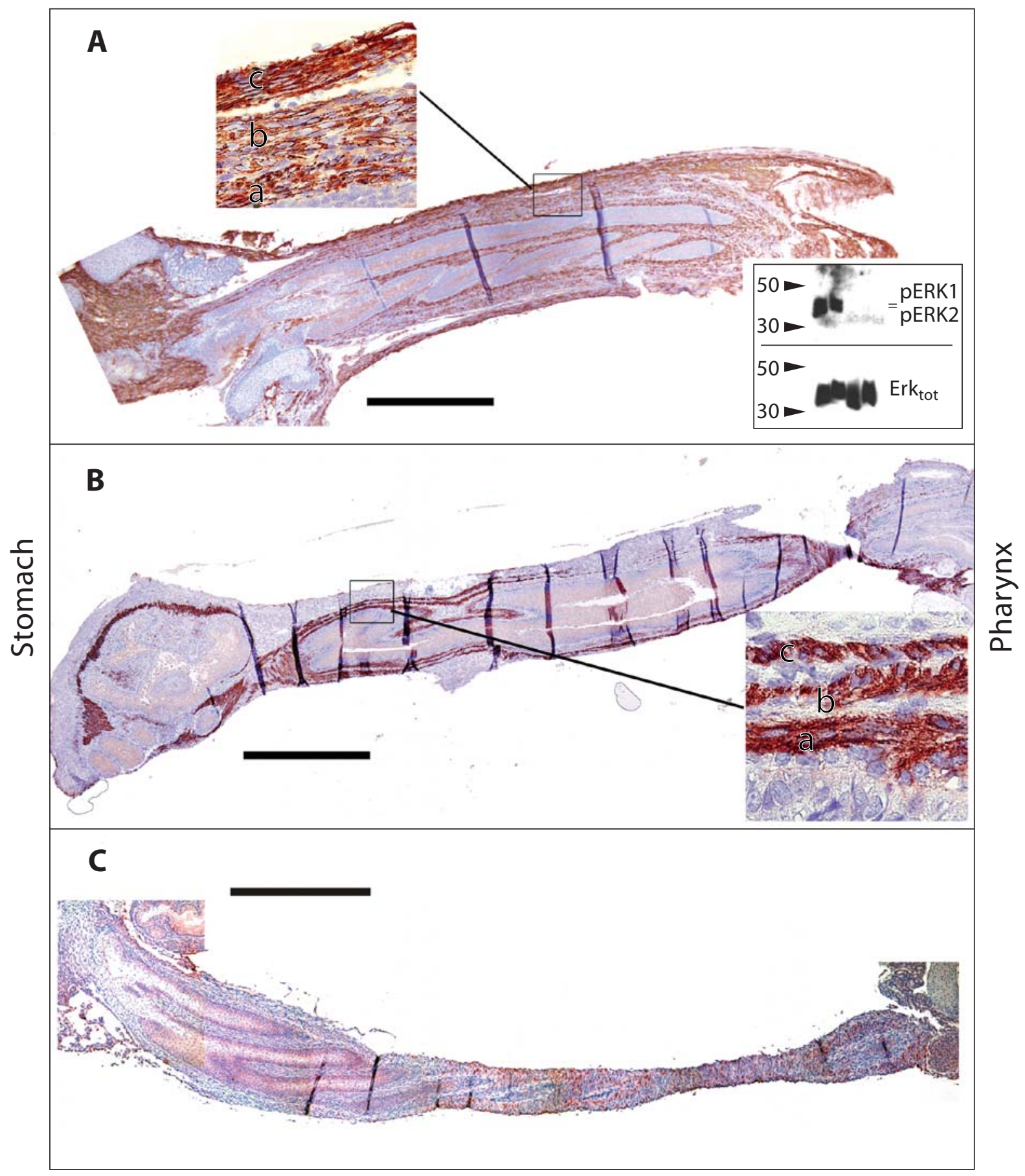

Fig. 4. Smooth muscle maintains smooth muscle actin expression in culture for 7 days, but is attenuated by FGF-2. A Sagittal section of E14.5 esophagus after 7 days of culture shows robust expression of smooth muscle actin in all 3 smooth muscle layers of the esophagus $(\mathrm{a}=$ muscularis mucosa, $\mathrm{b}=$ muscularis externa inner layer, $\mathbf{c}=$ muscularis externa outer layer). B Smooth muscle actin expression is attenuated after 7 days of culture when FGF-2 is added to the culture media (a muscularis mucosa, $\mathbf{b}$ muscularis externa inner layer, c muscularis externa outer layer). Note the loss of actin in the outer gastric muscle layers. C Myogenin expression remains unaffected by culture in FGF-2. Scale bars $=500 \mu \mathrm{m}$. Inset PD98059 inhibits ERK1/2 phosphorylation by FGF-2. E14.5 esophageal lysates, immunoblotted with anti-phospho-ERK1/2 or anti-pan-ERK antibodies, show complete inhibition of ERK1/2 phosphorylation by FGF-2 after concurrent treatment with MEK inhibitor PD98059 (upper panel, lanes 3 and 4). Esophagi treated with FGF-2 alone show robust phosphorylation of ERK1 and ERK2 (upper panel, lanes 1 and 2). Total ERK expression is similar for all samples (lower panel). 
and myogenin [Weyman and Wolfman, 1998; Jones et al., 2001; Tortorella et al., 2001]. Similarly, SMCs cultured in FGF-2 undergo dedifferentiation or fail to express SMC contractile proteins [Thyberg, 1996; Kato et al., 1998; Kawai-Kowase et al., 2004]. To determine if esophageal striated myogenesis was modulated through an FGF-2dependent pathway, esophageal explants were cultured in $25 \mu \mathrm{g} / \mathrm{ml} \mathrm{hrFGF-2} \mathrm{for} 7$ days. Under these conditions spontaneous peristalsis was markedly diminished over the culture period. A decrease in both frequency and amplitude of peristaltic waves was first observed after 3 days of culture, with FGF-2-treated esophagi contracting every 45-60 s, compared to control esophagi that contracted every 20-30 s. Inhibition of MEK with PD98059 prevented this attenuation of contractility.

Histologically, $\alpha$-smooth muscle actin expression decreased in the muscularis externa after 7 days of culture in FGF-2 augmented media (fig. 4B), consistent with the observed loss of function. Inhibition of MEK with PD98059 resulted in attenuated phospho-ERK1/2 expression (fig. $4 \mathrm{~A}$, inset) and prevented the decrease in $\alpha$-smooth muscle actin expression, consistent with previous observations [Kawai-Kowase et al., 2004]. However, striated myogenesis continued in the presence of FGF-2, with little or no attenuation of myogenin expression (fig. 4C). These findings suggest that myogenin expression may not be FGF-2 dependent in the esophagus or in vivo and may be a phenomenon observed only in cell culture. This is consistent with observations that mice overexpressing FGF-2 develop normal striated appendicular muscle, although in that study the FGF-2 may have been unable to interact with FGF receptors [Coffin et al., 1995].

\section{c-kit-Neutralizing Antibody, ACK2, Fails to Inhibit Peristalsis in Cultured Fetal Esophagi}

To determine if the peristalsis was induced by c-kitpositive ICC, we immunostained esophageal and gastric sections from 14.5-day-old uncultured fetuses with an anti-CD117 antibody. We could detect occasional immunopositive cells within the esophageal and gastric muscularis externa. Based on previous studies that documented inhibition of peristalsis in cultured fetal ureters after c-kit blockade [David et al., 2005], we cultured fetal esophagi in media containing $5 \mu \mathrm{g} / \mathrm{ml}$ ACK-2 c-kit-neutralizing antibody. This failed to inhibit peristalsis over the 7-day culture period, despite strong penetration of the antibody into the muscularis externa (fig. 5), indicating that spontaneous peristalsis within the fetal esophagus is not ICC-dependent.

Myogenesis in Fetal Esophageal Culture
Fig. 5. ACK2 antibody penetrates esophagi in culture but fails to inhibit peristalsis. Sagittal section of E14.5 esophagus labeled with a biotinylated goat anti-rat antibody and detected with Texas Red-avidin. Fluorescence can be detected throughout the esophagus, indicating complete penetration of the antibody during the culture period.

\section{Discussion}

We have provided additional evidence that esophageal striated myogenesis occurs from a resident population of myogenic precursors and that the striated myogenic program may be resistant to FGF-2 inhibition. While we could not determine the early embryonic origin of esophageal StMC, our data support recently published studies showing resident myogenic precursors [Wörl and Neuhuber, 2005] and suggest that the progression of myogenesis depends on local factors. Additionally, we have, for the first time, cultured fetal murine esophageal explants, and maintained a functional phenotype for 7 days. The contractile behavior of explanted esophagi appears to be independent of ICCs.

Myogenin expression proceeded ex vivo in a rostrocaudal direction (from pharynx to stomach), consistent with previous observations that striated myogenesis is initially detected in the subpharyngeal regions and proceeds caudally along the length of the esophagus [Zhao and Dhoot, 2000a, b]. Expression of myogenin at day E14.5 was absent in any part of the esophagus, but progressed rostrocaudally over the 7 days in culture, similar to previous observations [Zhao and Dhoot, 2000c]. Similarly, the finding that myogenic precursors exist along $70 \%$ of the length of the esophagus by E14.5 is consistent with previous observations that Myf5 and MyoD expression was observable along most of the esophagus by this stage of development [Zhao and Dhoot, 2000a]. Our study demonstrates that these precursors did not migrate from a subpharyngeal location to the distal esophagus, but were resident in that tissue by E14.5. Had these precursors migrated from the subpharyngeal region along a smooth muscle lattice, mechanical disruption of the lat- 
tice should have prevented myogenin from being expressed in the distal segment. However, we found robust expression in partially disrupted esophagi, indicating that myogenic precursors were present in the distal esophagus prior to mechanical disruption. We cannot, however, rule out the possibility that these precursor cells migrate through the esophagus prior to E14.5. On the other hand, complete separation of the esophagi into two independent segments resulted in failure of myogenin expression in the distal (pregastric) segment, suggesting that either humoral or mechanical factors are involved in propagating a wave of myogenic activation and myodifferentiation. Additionally, we cannot conclude that the myodifferentiation observed ex vivo precisely mirrors events that occur in vivo. For example, cultured fetal esophagi failed to elongate appreciably over the 7-day culture period, whereas our previous studies of esophageal development in vivo demonstrate that the esophagus grows in length during the last week of gestation [Rishniw et al., 2007]. It is likely that mechanical factors (distraction of the esophagus by a growing thorax), which are absent in our culture model, contribute to the normal development of the esophagus.

The persistence of esophageal peristalsis in the presence of the c-kit-neutralizing antibody, ACK2, differs from the results obtained with fetal ureteral explants, where peristalsis was abolished within a few days of culture [David et al., 2005]. While we identified occasional c-kit-positive cells in E14.5 esophagi, and ACK2 penetration into tissues appeared sufficient to inhibit c-kit receptors, peristalsis remained unaffected. This suggests that peristalsis is propagated in explanted esophagi by spontaneous smooth muscle depolarizations, rather than ICCs.

Peristalsis occurred both antegrade and retrograde in explanted esophagi. Peristalsis in adults is predominant- ly antegrade, but retrograde peristalsis can occur (eructation and regurgitation in ruminants and birds). The direction of peristalsis is dependent, in part, on the site of initiation - in adults, swallowing initiates antegrade peristalsis. In our fetal culture experiments, gastric peristalsis appeared to trigger many of the retrograde peristaltic waves. Whether this occurred because of a continuity of electrical coupling between the stomach and esophagus that later disappears or was due to mechanical initiation of a peristaltic wave (stretch-induced depolarization of esophageal muscle) is not known. Furthermore, whether bidirectional peristaltic activity occurs in utero in mice is unknown.

The failure of FGF-2 to inhibit myogenin expression was unanticipated. An effect of FGF-2 was observed, namely, a dedifferentiation of SMCs (characterized by loss of smooth muscle actin) and a concomitant attenuation of spontaneous contractile function, indicating that FGF-2 concentrations in the media were sufficient to produce a physiological effect. However, myogenin expression continued unabated in the presence of FGF-2, suggesting that in this system, striated myogenesis is not completely inhibited by FGF-2.

In conclusion, we have developed an ex vivo fetal esophageal culture model that remains functional for at least 7 days. Striated myogenesis in this model proceeds with a rostrocaudal activation of resident myogenic precursor cells that are resistant to FGF-2 inhibition of differentiation, but are dependent on structural integrity of the esophagus.

\section{Acknowledgments}

This study was supported in part by NIH grant RO1 DK065992 (M.I. Kotlikoff) and NIH training grant T32RR07059-09 (M. Rishniw).

\section{References}

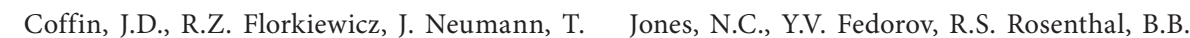
Mort-Hopkins, G.W. Dorn 2nd, P. Lightfoot, R. German, P.N. Howles, A. Kier, B.A. O’Toole (1995) Abnormal bone growth and selective translational regulation in basic fibroblast growth factor (FGF-2) transgenic mice. Mol Biol Cell 6: 1861-1873.

David, S.G., C. Cebrian, E.D. Vaughan Jr., D. Herzlinger (2005) c-kit and ureteral peristalsis. J Urol 173: 292-295. Physiol 186: 104-115.

Kablar, B., S. Tajbakhsh, M.A. Rudnicki (2000) Transdifferentiation of esophageal smooth to skeletal muscle is myogenic bHLH factordependent. Development 127: 1627-1639.

Kato, S., A. Muraishi, T. Miyamoto, J.C. Fox (1998) Basic fibroblast growth factor regulates extracellular matrix and contractile protein expression independent of proliferation in vascular smooth muscle cells. In Vitro Cell Dev Biol Anim 34: 341-346.
} 
Kawai-Kowase, K., H. Sato, Y. Oyama, H. Kanai, M. Sato, H. Doi, M. Kurabayashi (2004) Basic fibroblast growth factor antagonizes transforming growth factor-betal-induced smooth muscle gene expression through extracellular signal-regulated kinase 1/2 signaling pathway activation. Arterioscler Thromb Vasc Biol 24: 1384-1390.

Patapoutian, A., B.J. Wold, R.A. Wagner (1995) Evidence for developmentally programmed transdifferentiation in mouse esophageal muscle. Science 270: 1818-1821.

Rishniw, M., P.W. Fisher, R.M. Doran, E. Meadows, W.H. Klein, M.I. Kotlikoff (2007) Smooth muscle persists in the muscularis externa of developing and adult mouse esophagus. J Muscle Res Cell Motil 28: 153165.
Rishniw, M., H.B. Xin, K.Y. Deng, M.I. Kotlikoff (2003) Skeletal myogenesis in the mouse esophagus does not occur through transdifferentiation. Genesis 36: 81-82.

Stratton, C.J., Y. Bayguinov, K.M. Sanders, S.M. Ward (2000) Ultrastructural analysis of the transdifferentiation of smooth muscle to skeletal muscle in the murine esophagus. Cell Tissue Res 301: 283-298.

Thyberg, J. (1996) Differentiated properties and proliferation of arterial smooth muscle cells in culture. Int Rev Cytol 169: 183-265.

Tortorella, L.L., D.J. Milasincic, P.F. Pilch (2001) Critical proliferation-independent window for basic fibroblast growth factor repression of myogenesis via the p42/p44 MAPK signaling pathway. J Biol Chem 276: 1370913717.

Weyman, C.M., A. Wolfman (1998) Mitogen-activated protein kinase kinase (MEK) activity is required for inhibition of skeletal muscle differentiation by insulin-like growth factor 1 or fibroblast growth factor 2. Endocrinology 139: 1794-1800.
Wörl, J., W.L. Neuhuber (2005) Ultrastructural analysis of the smooth-to-striated transition zone in the developing mouse esophagus: emphasis on apoptosis of smooth and origin and differentiation of striated muscle cells. Dev Dyn 233: 964-982.

Zhao, W., G.K. Dhoot (2000a) Both smooth and skeletal muscle precursors are present in foetal mouse oesophagus and they follow different differentiation pathways. Dev Dyn 218: 587-602.

Zhao, W., G.K. Dhoot (2000b) Development and composition of skeletal muscle fibres in mouse oesophagus. J Muscle Res Cell Motil 21: $463-473$.

Zhao, W., G.K. Dhoot (2000c) Skeletal muscle precursors in mouse esophagus are determined during early fetal development. Dev Dyn 219: 10-20. 